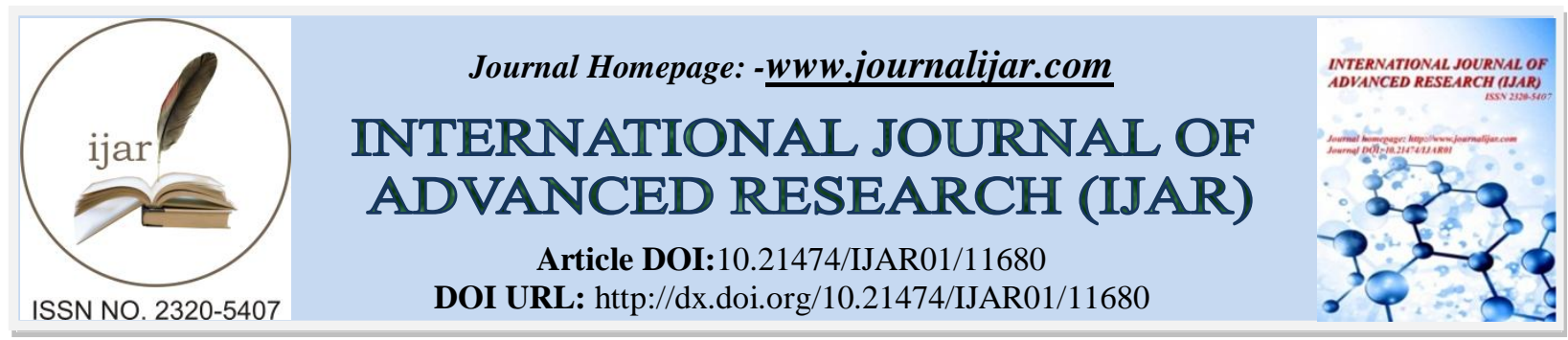

RESEARCH ARTICLE

\title{
APPLICATION OF BIO-SPECKLES FORDETECTION OF BIODEGRADATION OF PLASTIC POLYMERS BY THE BASIDIOMYCETOUS MUSHROOM HERICIUM ERINACEUS
}

\author{
O.A.Abd El Gaffar ${ }^{1}$, Y.M. Elsaba ${ }^{2}$ and H. El Ghandoor ${ }^{3}$ \\ 1. Departmentof Physics, Faculty of Science, Helwan University, Cairo, Egypt. \\ 2. Department of Botany and Microbiology, Faculty of Science, Helwan University, Cairo, Egypt. \\ 3. Department of Physics, Faculty of Science, Ain Shams University, Cairo, Egypt.
}

\section{Manuscript Info}

Manuscript History

Received: 10 July 2020

Final Accepted: 14 August 2020

Published: September 2020

Keywords:

Bio-Speckles,Image Processing

Contrast, Hericiumerinaceus,

Plastic Polymers,

\begin{abstract}
The wastes of solids like plastic form a huge pollutionproblem in the environment. Thus, this study is concerned about the use of fungi for biodegradation of plastic. The biodegradationcapability was assessed by growing Hericiumerinaceus mushroom on different types of plasticpolymer. The laser speckle measurements can detect the polymer degradation optically. This is done, when a coherent light is allowed to be incident on bio samples surfaces, such surfaces are considered optically rough. The scattered light centers from these surfaces represented the growth of Hericiumerinaceusfungus on polymer sample. These scattering lights are coherent with respect to each other, leading to a precisefringprint of these biological tissues. The scattered light presented a particular intensity distribution, forming bio speckle. Digital images were taken, then by image processing, intensityand contrast were calculated for each bio sample.
\end{abstract}

Copy Right, IJAR, 2020, All rights reserved.

\section{Introduction:-}

Hericiumerinaceusis an edible and medicinal basidiomycetous mushroom belonging to the family Hericiaceae, order Russulales and class Agaricomycetes [1]. This mushroom is also known as Lion's Mane, Monkey's Mushroom, Bear's Head, Hog's Head Fungus, White Beard, Old Man's Beard, Pom Pom or Bearded Tooth in other parts of the world[2].Hericiumerinaceus has wide and important applications including, anti-hyperglycemic property [3], antibacterial activity [4], antioxidant activity, neurological activity[5] and anticancer activity [6].The mushroom is suitable for bioremediation like in sewage treatment in the pulp and paper industry [7]where it has a high enzymatic activity that facilitates its practical applications. There is an application of lion's mane mushroom culture for commercial scale production of such enzymes as $\alpha$-amylase, cellulase, $\beta$-glucosidase, laccase and xylanase [8].

When a coherent light ray is allowed to be incident on an optically rough object, it will scatter randomly through all directions. Interference of these scattered rays leads to the formation of a bright spots (constructive interference), and dark spots (destructive interference), these are called Laser Speckles. The way in which the intensity of the scattered light varies in space depends on the shape of illuminating wavefront, on the material of the object as well as on the shape and surface finish. The rough objects or diffuse objects are usually of two types: diffuse reflecting objects and transparent diffuse objects, the rough surfaces of stones, cement, unpolished metal, wood, and so on are 'Diffuse reflecting objects. Ground glass is a well-known example of a Transparent diffuse object. It is difficult to

Corresponding Author:- O.A. Abd El. Gaffar

Address:- Departmentof Physics, Faculty of Science, Helwan University, Cairo, Egypt. 
determine a lower limit under which an object is considered well-polished, because it is not only the thickness variations that count, but their spatial distribution: in other terms, the slopes of the profile of the diffuse object.

Bio specklesare formed, when the bio medium of asample is illuminated with light having a high degree of coherence, such as laser light, the scattered light presents a particular intensity distribution, making the sampleappear to be covered with a fine granular structure. This structure, which consists of alternating dark and bright spots of varying shapes, and distributed in a random way as shown in figure 1, such intensity distribution, which is also observed when coherent light propagates through amedium presenting random variation in its refractive index, is known as a speckle pattern. Speckle patterns fill the space surroundingthe diffusive medium, and so they can be registered by properly placing CCD detector at a certain distance from the sample. It isusual to call speckle patterns obtained from free space propagation of light objectivespeckles, whereas those patterns obtained under image formation conditions arereferred to as subjective speckles.

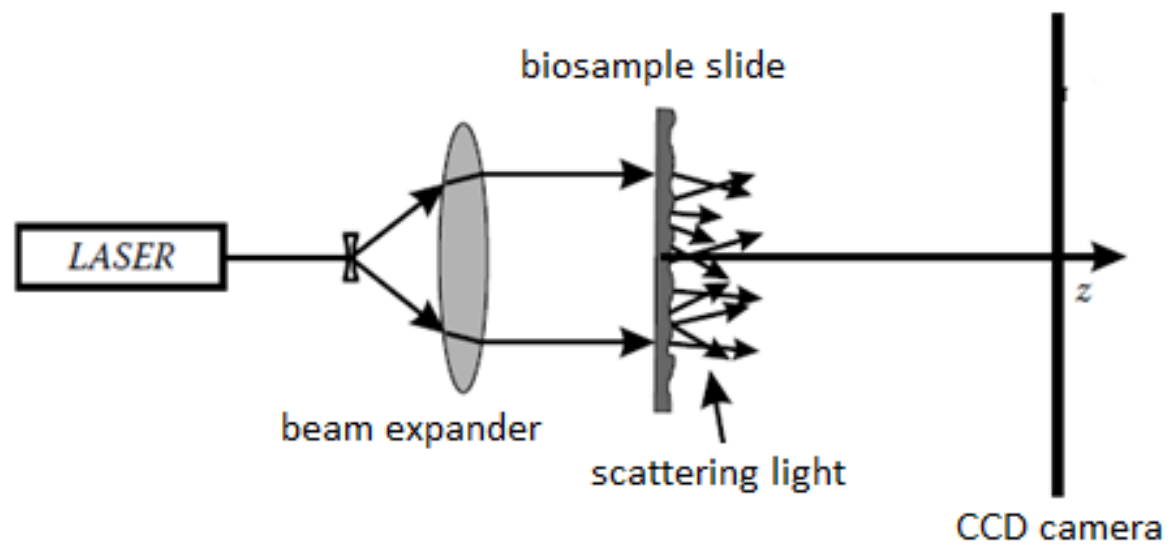

Fig. 1:- Transmission of coherent light through diffusive medium.

The statistical properties [9-12] of speckle patterns carry useful information about the biological samples such as size, intensity, and contrast which can be measured. In our work experiments, we use transmission speckle configuration to apply Bio speckles measurements which mentioned, as shown infigure 1.

\section{Theory: -}

When the specimen surface of bio sample is illuminated by a coherence laser beam (Fig. 1), the diffused light gives a granular pattern, because of the random spatial variation of intensity. This phenomenon is called speckle. The speckle pattern can be formed either by free-space propagation or from an imaging system.

The average size $\left\langle\sigma_{0}>\right.$ of objective speckles is related to the wavelength of light $\lambda$ and to the angle subtended by the scattering area on the screen. For a circular illuminated area of diameter D, the relationship is given by:

$<\sigma_{0}>=\frac{1.22 \lambda \mathrm{L}}{\mathrm{D}}$

Where speckle pattern formed at a distance $\mathrm{L}$ from the scattered surface that is uniformly illuminated by a coherent beam. While the minimum size $\rho_{\mathrm{s}}$ of the image speckles is related to the imaging system $\mathrm{f}$-number $\mathrm{F}$ and the magnification $\mathrm{M}$, and is given as:

$\rho_{\mathrm{s}}=1.2(1+\mathrm{M}) \lambda \mathrm{F}$

Where $\lambda$ is the wavelength of the laser, and $\rho_{\mathrm{s}}$ is the radius of the Airy disc that is formed for the given optical imaging configuration figure (2).

Generally, the statistical properties of the speckle pattern depend upon both the coherence of the incident light and the detail properties of the scattering surface.

The average contrast of the image, either acquired as a bitmap or extracted from the frames of the movie, is currently calculated $[9,13]$ as 


$$
\mathrm{C}=\frac{\left\langle\left(\mathrm{I}(\mathrm{i}, \mathrm{j})-\langle\mathrm{I}\rangle^{2}\right)\right\rangle^{\frac{1}{2}}}{\langle\mathrm{I}\rangle}
$$

$$
\operatorname{orC}=\frac{\sigma}{\langle\mathrm{I}\rangle}
$$

where $I(i, j)=I\left(x_{i}, y_{j}\right)$ is the intensity recorded by the cell $(i, j)$ of the digital camera, hence by the pixel $(i, j)$ of the array of pixels the image consists of. This is a space contrast and not a time contrast, as pointed out in [14]. The contrast definesthe ratio of the standard deviation $\sigma$ of the average intensity I, where the brackets denote spatial averaging intensity.

In this work, the image contrast is calculated with the related equation:

$$
\mathrm{C}=\frac{\mathrm{I}_{\max }-\mathrm{I}_{\min }}{\mathrm{I}_{\max }}
$$

where $I_{\max }$ is the average of the maximum intensity (bright grains) and and $I_{\min }$ is the average of the minimum intensity (dark grains).

\section{Experimental setup and data processing procedure: -}

Plastic polymerdegradationandHericiumerinaceus: -

The fungus used in this study was Hericiumerinaceusmushroom which obtained from the mycology unit, Desert Research Center, Cairo, Egypt. The fungus was preserved on potato dextrose agar (PDA) slant [15] at $4^{\circ} \mathrm{C}$.

Hericiumerinaceuswas assessed for its ability to degrade different plastic polymers (Sigma Aldrich) including polyurethane (PUR), polyethylene (PE), polypropylene (PP), polyvinyl chloride (PVC) using modified method [16], by inoculating $0.5 \mathrm{~cm}$ fungal disc of 7 days old culture on solid medium containing g/l $\mathrm{K}_{2} \mathrm{HPO}_{4}, 0.5 ; \mathrm{KH}_{2} \mathrm{PO}_{4}, 0.04$; $\mathrm{NaCl}, 0.1 ; \mathrm{CaCl}_{2} . \mathrm{H}_{2} \mathrm{O}, 0.002 ;(\mathrm{NH} 4){ }_{2} \mathrm{SO}_{4}, 0.2 ; \mathrm{MgSO}_{4} .7 \mathrm{H}_{2} \mathrm{O}, 0.02 ; \mathrm{FeSO}_{4}, 0.001$, Agar 20.0. The media were supplemented with $10 \mathrm{~g}$ from each polymer separately.

\section{Slide preparation for examination: -}

Fungal tissue $(0.5 \mathrm{~cm})$ from the previously prepared plates of each tested polymer was put on a clean slide for examination to show the degrading effect of the fungus on different tested plastic polymers compared to the control (agar medium without any plastic polymer).

\section{Set up the formation of bio speckle: -}

The incident light from coherence laser strikes the microscope objective lensL $\mathrm{L}_{1}$ of focal lens $5 \mathrm{~mm}$ to expand the laser beam. The expanded laser beam is then incident on a collimating lens, where aplane coherent wave was obtained. These collimated coherent beams of green wavelengths $532.0 \mathrm{~nm}$ with output power $<50 \mathrm{mWwere}$ used to illuminate a bio sample slide. The schematic of the experiment is presented in Figure2.A circular aperture at distance $\mathrm{D}=100 \mathrm{~mm}$ was used adjacent and parallel to a lens plane $\mathrm{L}_{2}$ of focal length $\mathrm{f}=150 \mathrm{~mm}$. A high-resolution CCD camera (charge-coupled-device) was used to record the image of the resultant structure of bio speckle digitally. Measurements were done atan angle $\theta$ degrees from the beam axis using a CCD camera. The procedure that was used in this work is a part of the procedure described in $[17,18]$ primarily because it is a lower cost procedure. Then data of the images were processing.

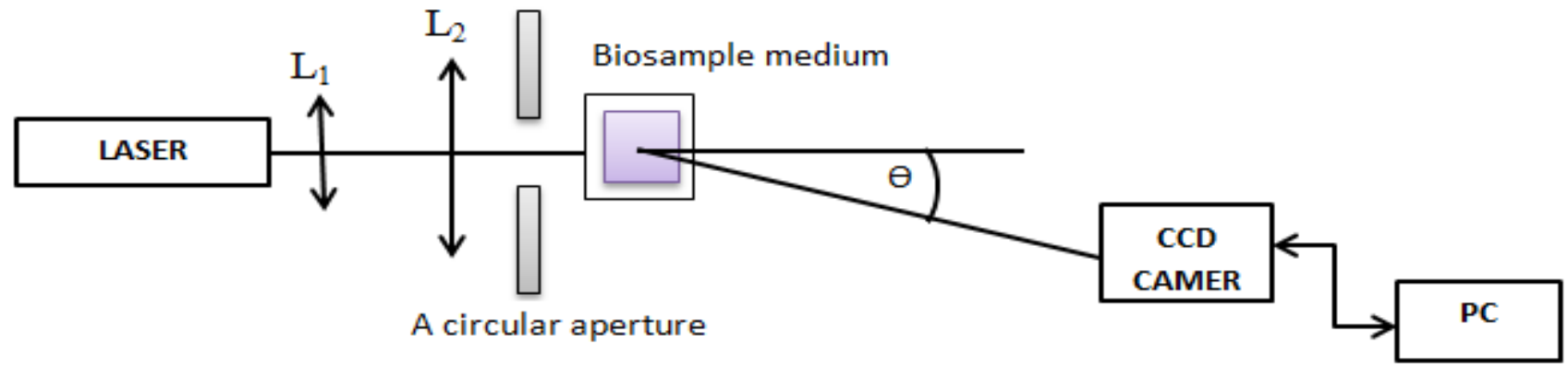

Fig.2:-Bio speckle pattern produced when the sample is imaged on the CCDcamera through an optical system. 
In this paper, the mushroom fungus Hericiumerinaceus grown on different types of polymers such as PP, PU, PE, and PVC. Each type of polymer sample used has different values of particle size, density and refractive index as shown in table1[ 19,20]. In addition, the used physical properties of Hericiumerinaceus is also shown in table 2.[21,22]

Table 1:-Table of the usedphysical properties of polymers.

\begin{tabular}{|l|c|c|}
\hline Plastic polymer & $\begin{array}{c}\text { Particle size (minimum diameter) } \\
(\boldsymbol{\mu m})\end{array}$ & $\begin{array}{c}\text { Density } \\
\left(\mathbf{1 0 ^ { 3 }} \mathbf{~ k g} / \mathbf{m}^{3}\right)\end{array}$ \\
\hline Polyethylene (PE) & $\mathbf{2 3 - 9 0}$ & $\mathbf{0 . 0 3}$ \\
\hline polypropylene (PP) & $\mathbf{3 6}-\mathbf{1 2 0}$ & $\mathbf{0 . 9 2 5}$ \\
\hline polyvinyl chloride (PVC) & $\mathbf{7 1 - 1 1 0}$ & $\mathbf{1 . 4 0 5}$ \\
\hline polyurethane (PU) & $\mathbf{1 4 - 8 0}$ & $\mathbf{0 . 9 3 5}$ \\
\hline
\end{tabular}

Table 2:-Physical properties ofHericiumerinaceus.

\begin{tabular}{|c|c|c|}
\hline $\begin{array}{c}\text { (hericiumerinaceus) } \\
\text { fungi }\end{array}$ & $\begin{array}{c}\text { Particle size (minimum diameter) } \\
(\mu \mathrm{m})\end{array}$ & $\begin{array}{c}\text { Density } \\
\left(10^{3} \mathrm{~kg} / \mathrm{m}^{3}\right)\end{array}$ \\
\hline control medium without plastic & $4-6$ & $1.08-1.011$ \\
& $<5>1.095>$ \\
\hline
\end{tabular}

\section{Results and Discussion: -}

Plastic degradation byHericiumerinaceus was determined and the formation of a small halo around fungal disc in all tested plastic polymers as shown in figures (3-7) indicating the degrading ability of Hericiumerinaceus to all used polymers.

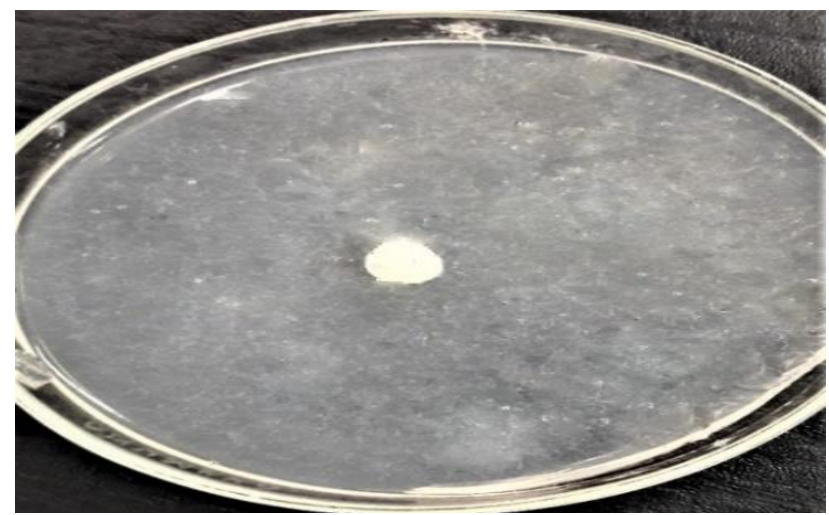

Fig.3:-Hericiumerinaceus grown on control medium without plastic after 14 days.

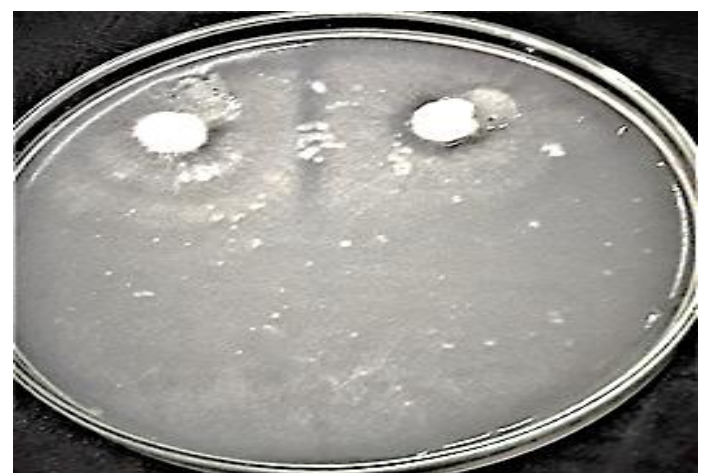

Fig. 4:-Hericiumerinaceus grown on Polyethelene (PE) agar medium after 14 days. 


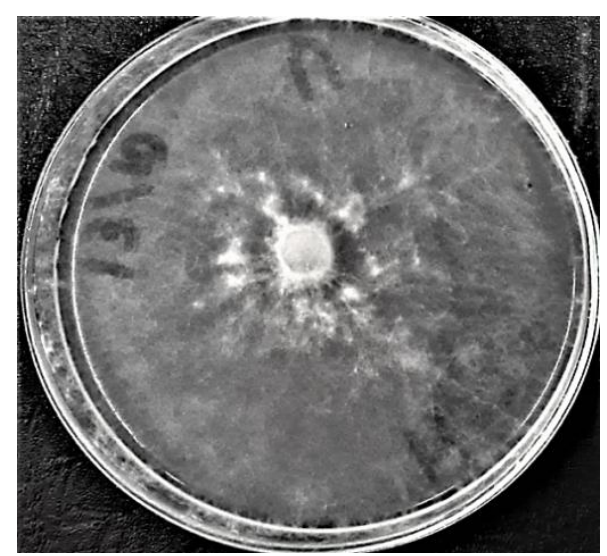

Fig. 5:-Hericiumerinaceus grown on Polyurethane (PU) agar medium after 14 days.

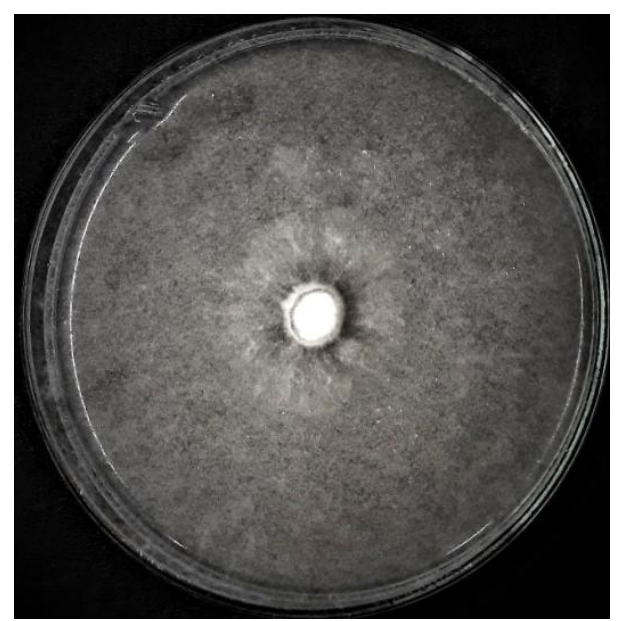

Fig. 6:-Hericiumerinaceus grown on polyvinylchloride (PVC)agar medium after 14 days.

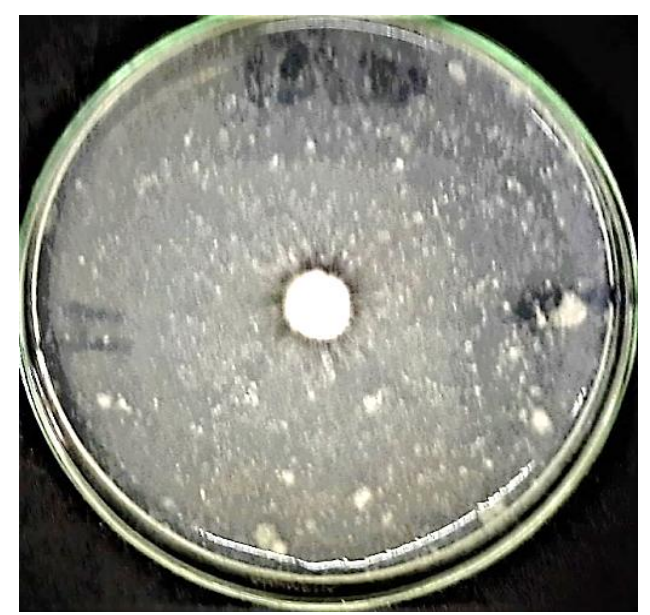

Fig. 7: -Hericiumerinaceus grown on Polypropylene (PP) agar medium after 14 days.

Slide examination of different bio sample types of Hericiumerinaceusgrown on different types of polymers, was investigated by the laser speckle patterns obtained using a coherent laser source of green wavelength as shown in figures 3(a, b), 4(a, b), 5(a, b), 6(a, b) and 7(a, b). 


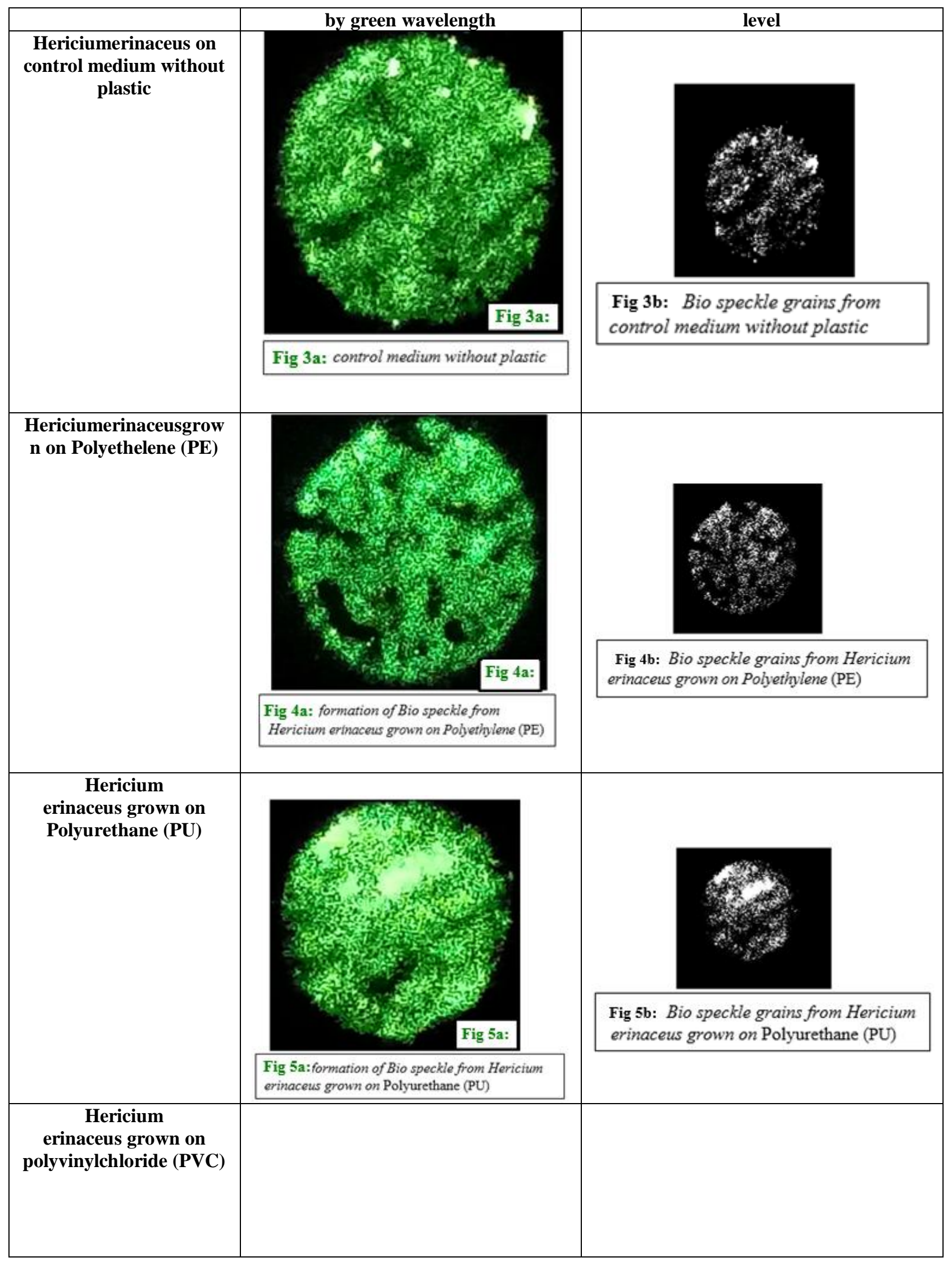




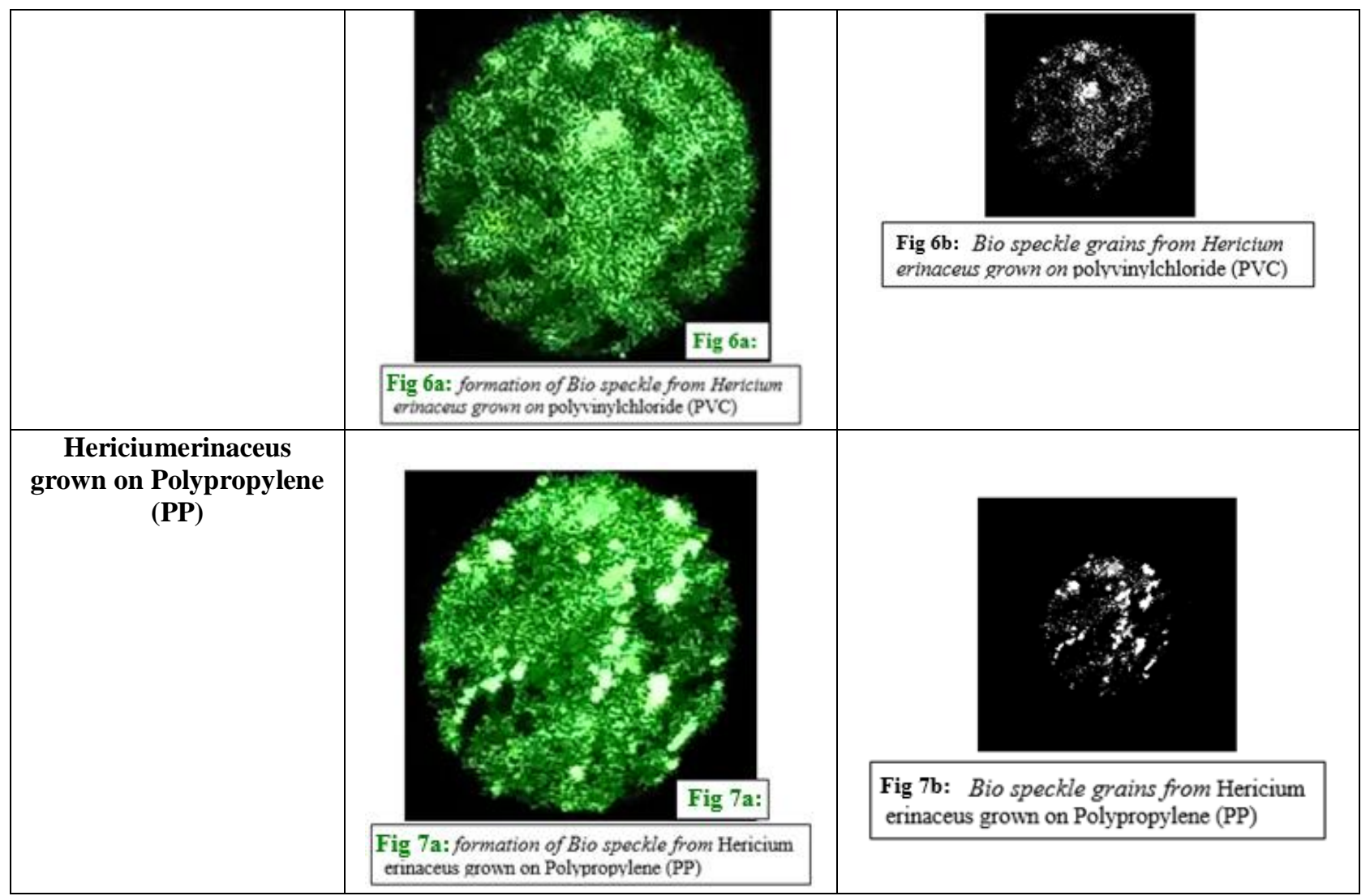

Digital image analysis

From figures shown below $(3 c, 4 c, 5 c, 6 c, 7 c)$, these results reveal about the speckle intensity fluctuationsfor all different bio sample types of Hericiumerinaceusgrown on different types of polymers which calculate the average contrast.

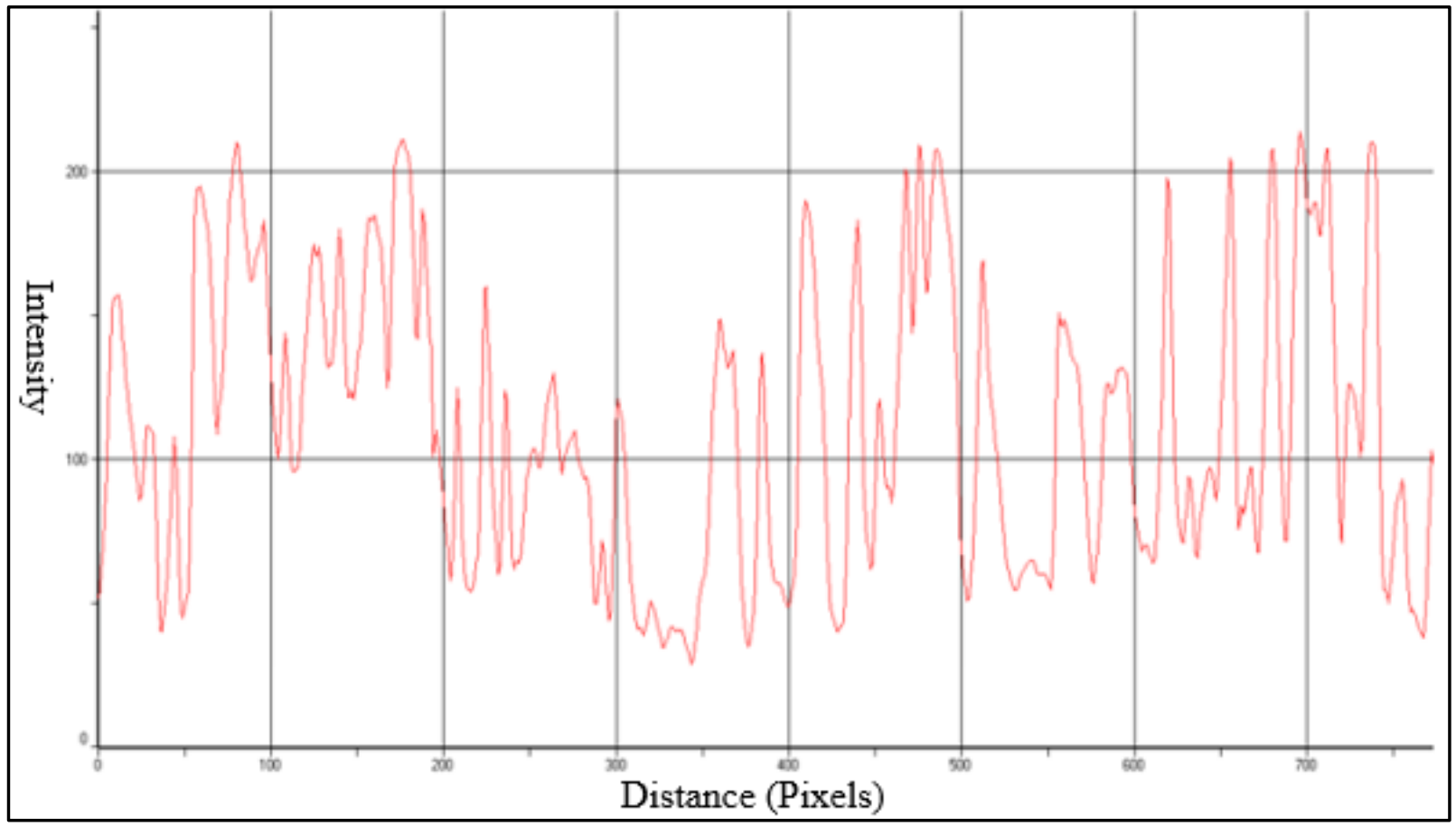

Fig 3c: - The intensity fluctuations of bio speckle from an image processing of control medium without plastic. 


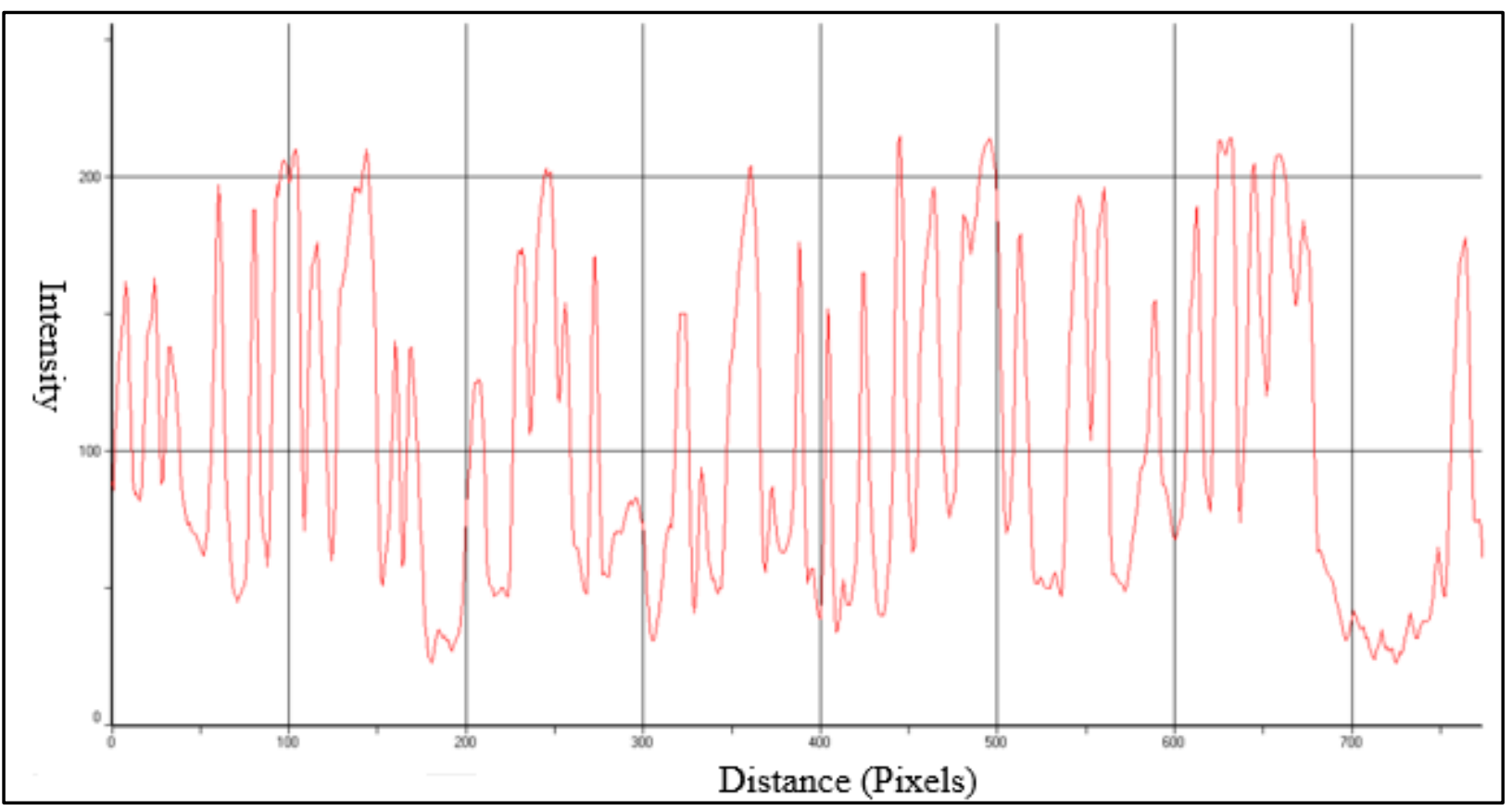

Fig 4c: - The intensity fluctuations of bio speckle from an image processingofHericiumerinaceusgrown on polyethylene $(\mathrm{PE})$.

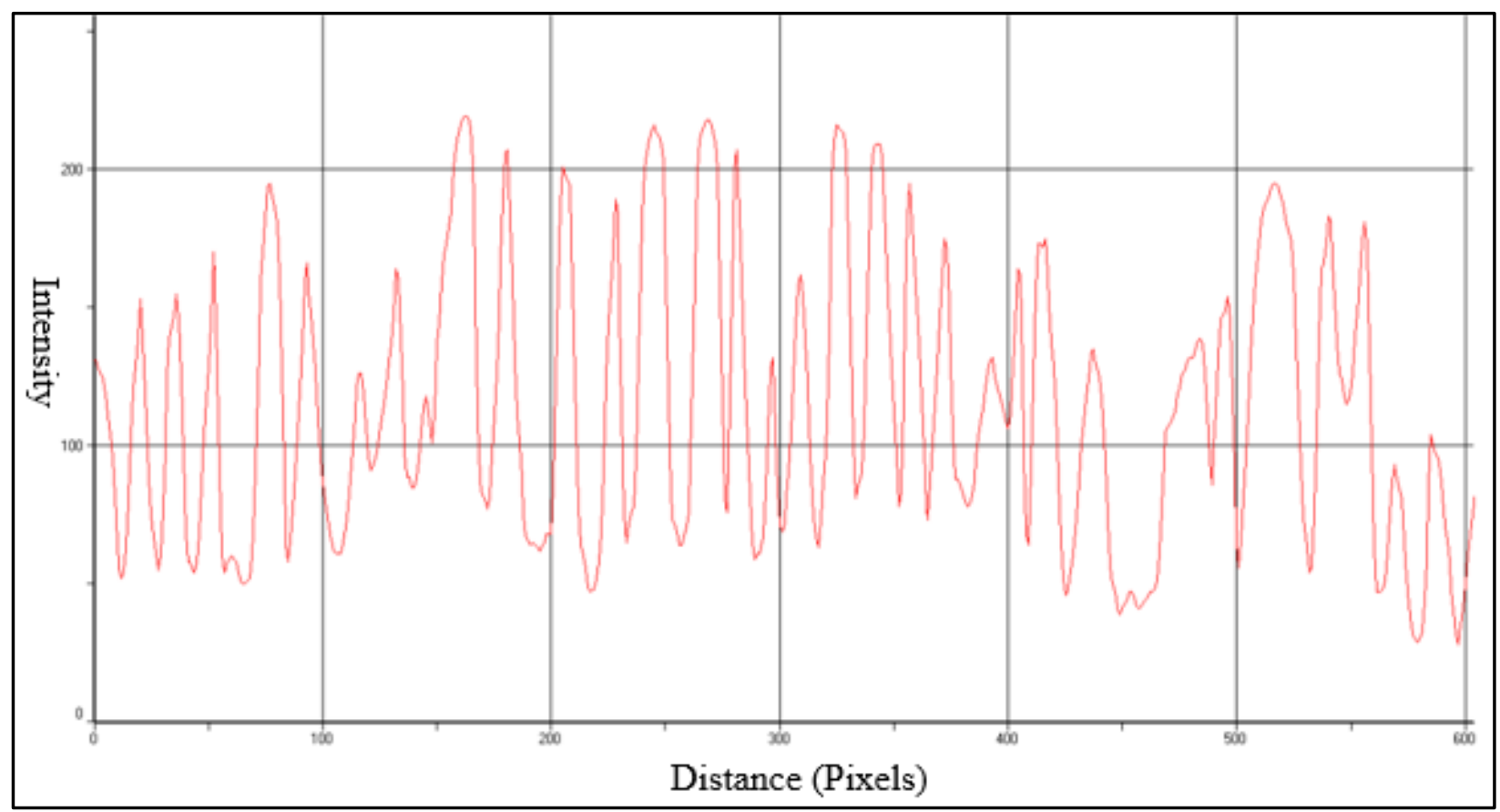

Fig 5c:-The intensity fluctuations of bio speckle from an image processingofHericiumerinaceus grown on Polyurethane (PU) 


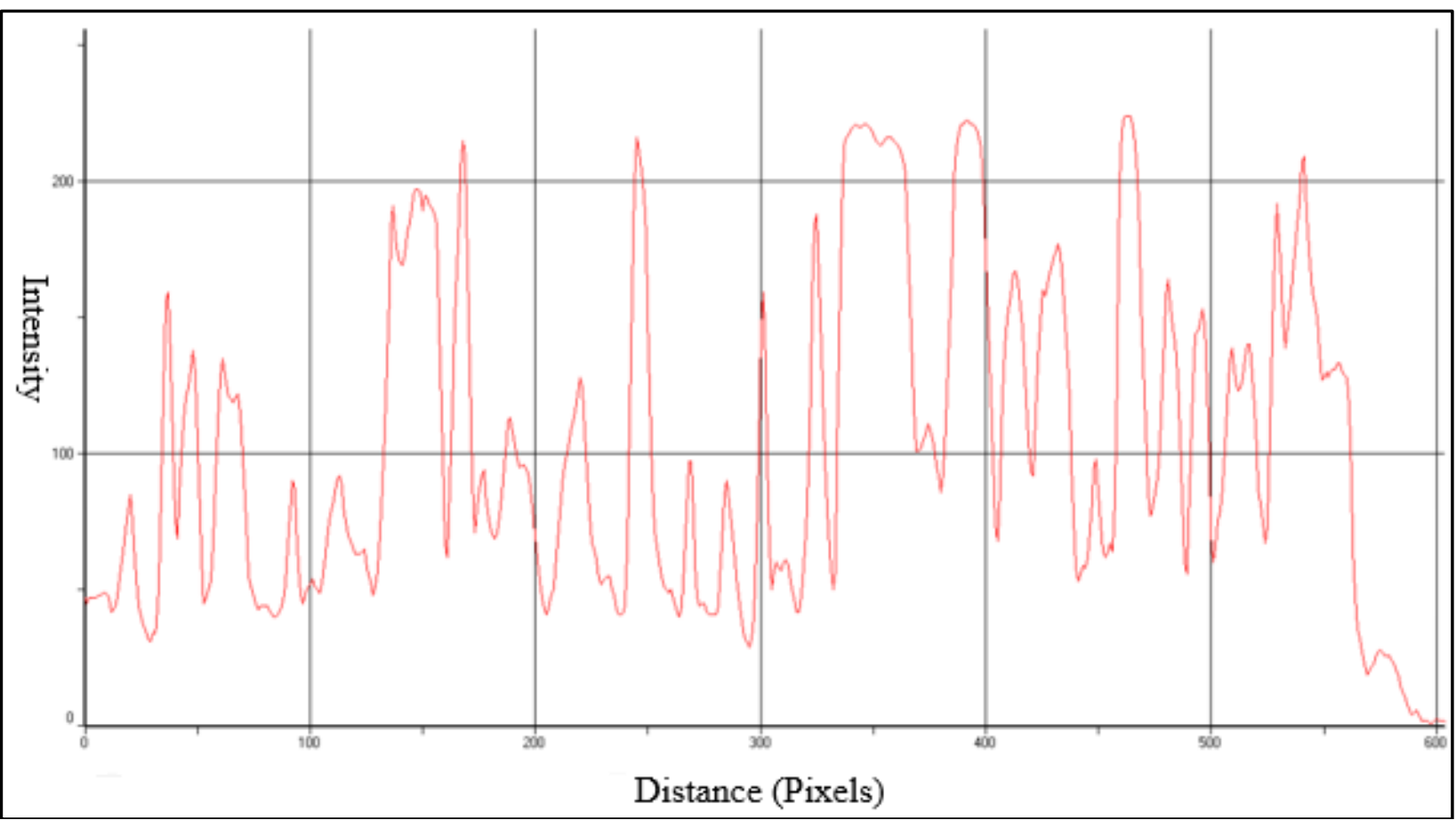

Fig 6c: - The intensity fluctuations of bio speckle from an image processing of Hericiumerinaceus grown on polyvinylchloride (PVC).

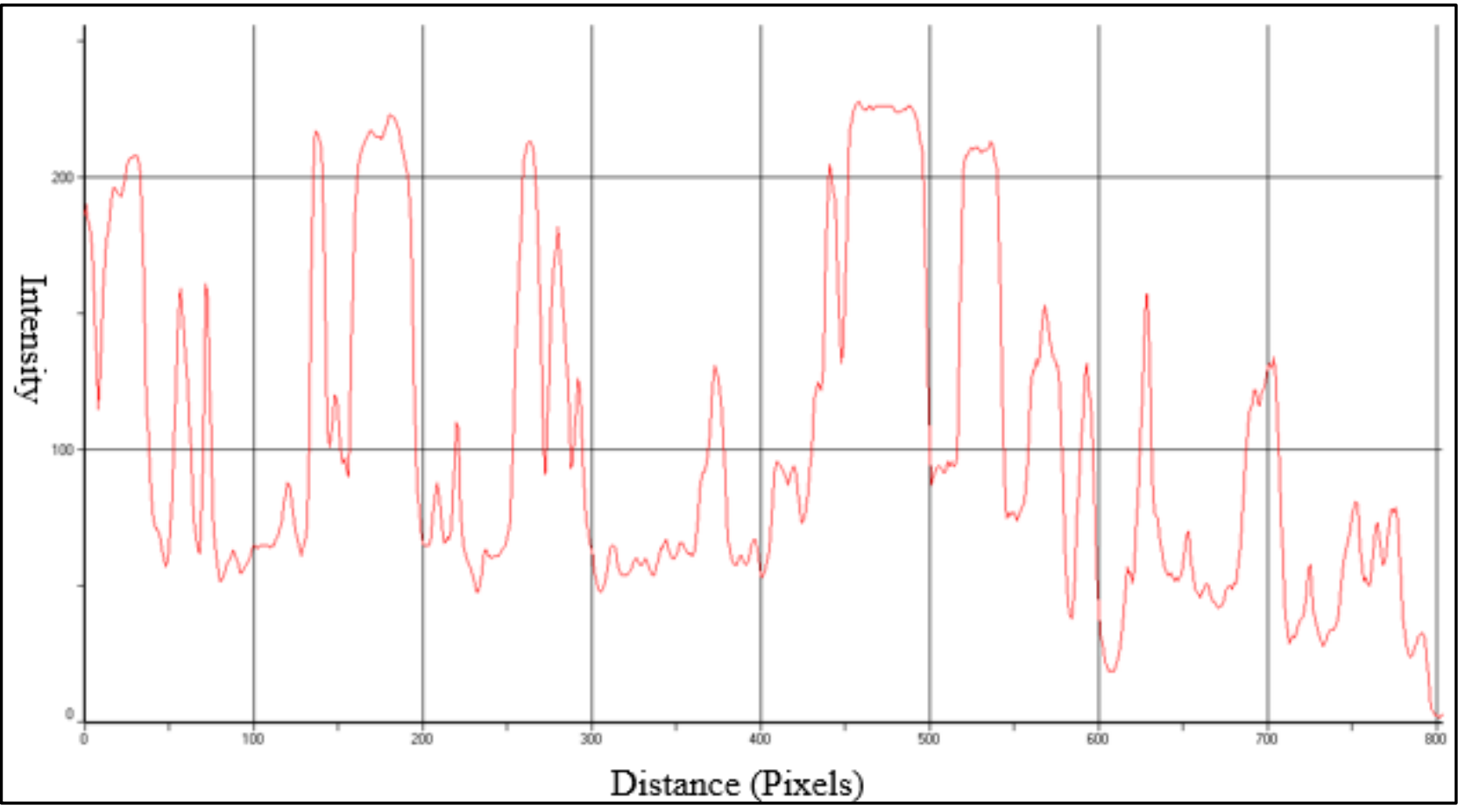

Fig 7c: -The intensity fluctuations of bio speckle from an image processing of Hericium erinaceous grown on Polypropylene (PP).

An average speckle contrast was calculated for every image according to the tested bio sample of polymer degradation, the relation between contrast and the different polymers degradation samples is shown in figure (8).

The graph between densities of polymers and Hericiumerinaceus with respect to the contrastin figure (9). 
Statistically, it shows that $\mathrm{R}^{2}$ measures the change of contrast values explained by independent densities of polymers and Hericiumerinaceus for a linear regression equation $y=a+b x$ and the computationalvalue of the adjusted $\mathrm{R}$-squared is (-0.0439).

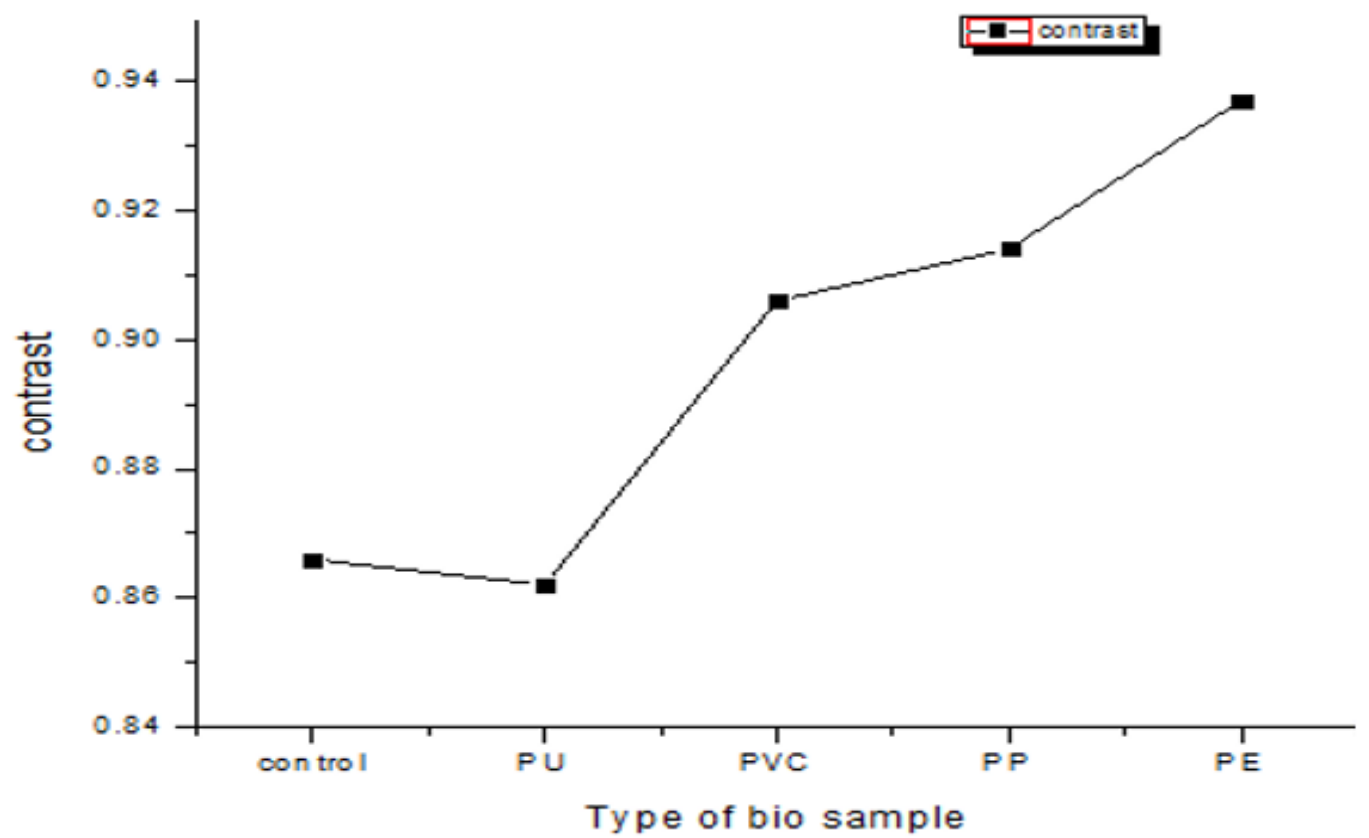

Fig.8: - The average contrast with the different bio polymer's degradation samples.

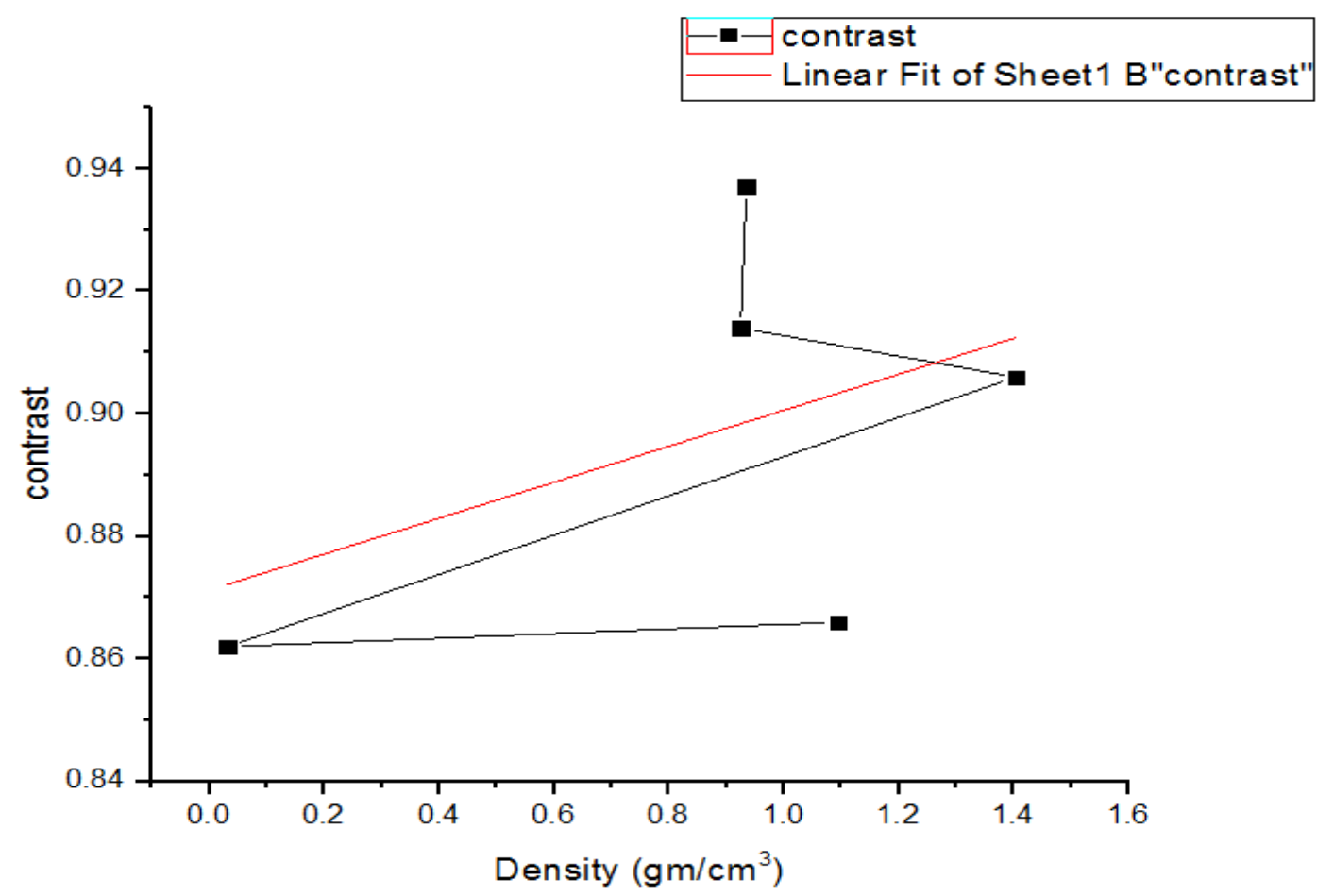

Fig.9: - The average contrast with densities of polymers and Hericiumerinaceus.

\section{Conclusion: -}

This study was the first report for the application of Bio-speckles for detection of biodegradation of plastic polymers by the basidiomycetous mushroom Hericiumerinaceus. The aim of this work is to accurately detect minute surface 
roughness of the scattered light centers from surfaces represented the growth of Hericiumerinaceusfungus on plastic polymeric samples. Accurate results for the evaluation of the growthofHericiumerinaceus fungus on plastic polymeric samples have been obtained based on the different texture features that have been extracted from the gray-level, laser speckle imaging technique based on second order statistics can be used as an additional tool for the evaluation of degradation polymers.

\section{References:-}

1. Kirk PM, Cannon PF, Minter DW, Stalpers JA (2008) Dictionary of the fungi. 10th ed., p. 313.

2. Thongbai, B., Rapior, S., Hyde, K. D., Wittstein, K. and Stadler, M.Hericium erinaceus, an amazing medicinal mushroom.Mycol Progress (2015) 14:91.DOI 10.1007/s11557-015-1105-4.

3. Chaiyasut, C. and Sivamaruthi, B.S. Anti-hyperglycemic property ofHericium erinaceus-A mini review /Asian Pac J Trop Biomed 2017; 7(11): 1036-1040

4. Shen, T. Morlock, G. and Zorn, H. Production of cyathane type secondary metabolites by submerged cultures of Hericium erinaceus and evaluation of their antibacterial activity by direct bioautography. Fungal Biol Biotechnol (2015) 2:8. DOI 10.1186/s40694-015-0018-y

5. Spelman, K., Sutherland, E. and Aravind, B. Neurological Activity of Lion's Mane (Hericium erinaceus). Journal of Restorative Medicine 2017; 6: page 19.

6. Younis, A.M. Anticancer potential of Hericium erinaceus extracts against particular human cancer cell lines. Microbial Biosystems 2017; 2(1): 9-20.

7. Wang MX, Guan D, Jing MD. Nutritional composition analysis of five strains of Hericium erinaceus and their utilization. Microbiology. 1992;19(2):68-72.

8. Ko HG, Park SH, Kim SH, Park HG, Park WM. Detection and recovery of hydrolytic enzymes from spent compost of four mushroom species. Folia Microbiol (Praha). 2005;50(2):103-106. http://dx.doi.org/10.1007/BF02931456

9. Goodman, J. W., Statistical properties of laser speckle patterns, in Laser Speckle and Related Phenomena, 2nd ed., Dainty, J. C., Ed., Springer Verlag, New York, 1984, chap. 2.

10. Goodman, J. W., Some fundamental properties of speckle, J. Opt. Soc. Am., 66, 1145,1976.

11. Dainty, J. C., Laser Speckle and Related Phenomena, 2nd ed., Dainty, J. C., Ed.,Springer Verlag, New York, 1984.

12. Dainty, J. C., The statistics of speckle patterns, in Progress in Optics, Wolf, E., Ed.,North-Holland, 1976, 14.

13. J.D. Briers, Physiol. Meas. 22, R35 (2001)

14. R. Nothdurft, G. Yao, Opt. Express 13, 10034 (2005)

15. Salfinger Y., and Tortorello M.L., 2015, Compendium of Methods for the Microbiological xamination of Foods, 5th Ed., American Public Health Association, Washington, D.C.

16. Russell JR, Huang J, Anand P, Kucera K et al. 2011 - Biodegradation of polyester polyurethane by endophytic fungi. Applied and environmental microbiology 77, 6076-6084.

17. Y. Piederri’ere, J. Cariou, Y. Guern, B. Le Jeune, G. Le Brun, J. Lotrian, Opt. Express 12, 176, (2004)

18. Y. Piederriere, J. Le Meur, J. Cariou, J.F. Abgrall, M.T. Blouch, Opt. Express 12, 4596, (2004)

19. Focal plane array detector-based micro-Fourier-transforminfrared imaging for the analysis of microplastics inenvironmental samples. Environ. Chem. 2015, 12, 563-581Martin Gu" nter Joachim Lo"der, MircoKuczera, Svenja Mintenig, ClaudiaLorenzand Gunnar Gerdts. Email: martin.loeder@uni-bayreuth.de

20. https://www.engineeringtoolbox.com/density-solids-d_1265.html

21. Maheshwari, R. (2016). Fungi: Experimental Methods in Biology, Second Edition. Mycology. CRC Press. p. 3. ISBN 978-1-4398-3904-1

22. Buoyant Densities and Dry-Matter Contents of Microorganisms: Conversion of a Measured Biovolume into Biomass. ARS R. BAKKEN* AND ROLF A. OLSENDepartment of Microbiology, Agricultural University of Norway, P.O. Box 40, N-1432 Aas-NLH, NorwayReceived 25 August 1982/Accepted 27 December 1982. 